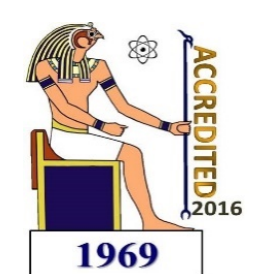

https://djs.journals.ekb.eg/

Research Article

MATHEMATICS

\title{
Solving a Fully Rough Integer Linear Fractional Programming Problem
}

\author{
Authors: El-Saeed Ammar , Tarek El jerbi
}

Affiliations: Department of mathematics, Faculty of science. Tanta University.

KEY WORDS

Integer

programming,

Fractional

programming, Integer linear fractional programming, Rough set theory, Rough integer interval.
ABSTRACT

In this paper, a fully rough integer linear fractional programming problem is introduced, in which all coefficients and decision variables in the objective function and the constraints are rough intervals. The optimal value of decision rough variables is rough interval. In order to solve this problem, we will construct four crisp integer linear fractional programming problems. Via these four crisp problems the rough optimal integer solution is obtained. An illustrative numerical example is given for the developed theory.

\section{Introduction}

The main interest in fractional programming was generated by the fact that a lot of optimization problem from engineering, natural resources and economics require the optimization between physical and / or economic functions. The problems, where the objective function is a ratio of two linear functions subject to a set of linear constraints and nonnegative integer variables constitute an integer linear fractional programming problem.
The integer solution of fractional programming problem is proposed [1]. Several methods were suggested for solving integer linear fractional programming problem such as variable transformation method, as well as branch and bound method [2].

Borza et al. [3]proposed the method to solve linear fractional programming problem with interval coefficients in objective function. Jayalakshmi and Pandian, Proposed a new 
method namely, denominator objective restriction method for finding an optimal solution to linear fractional programming problems [4]. Linear fractional programming problem with interval coefficients in the objective function is introduced [5], It is proved that we can convert an IVLFP to an optimization problem with interval valued objective function which its bounds are linear fractional functions. Rough Set Theory (RST) was initiated by Pawlak [6] in 1982 as a method for ambiguity management. Pandian et al. [7] considered that the transportation problem has all or some parameters as rough integer intervals. Also, proposed a new method named, a slice-sum method to solve Rough Integer Interval Transportation Problem (RIITP), where transportation cost, supply and demand are rough integer intervals. Hamazehee et al. [8] introduced a new class of Linear Programming (LP) problems in which some or all of the coefficients are rough intervals and showed that each one of them can be transformed into two LP problems with interval coefficients. Ammar and Muamer. [9] introduced a rough linear fractional programming problem. They are considered a rough interval in the objective function coefficient. Emam et al. [10] presented a solution of fully rough three level large scaler linear programming problem, in which all decision parameters and decision variables in the objective functions and the constraints are rough intervals. Algorithm for solving fuzzy rough linear fractional programming problems
(FRLFP) is introduced, All the variables and coefficients of the objective function and constraints are fuzzy rough number [11]. A Large-Scale three level fractional problem is introduced with random rough coefficient in the objective function in [12].

\section{BASIC PRELIMINARIES}

In this section a basic notions of interval analysis are given [5]:

Definition 2.1. Suppose $I$ is the set of all compact intervals in the set of all real numbers $R$. If $A \in I$ then we write $A=\left[a^{L}, a^{U}\right]$ with $a^{L} \leq a^{U}$ and the following holds:
i. $A \geq 0$ iff $a^{L} \geq 0$
ii. $A \leq 0$ iff $a^{U} \leq 0$

\subsection{Basic operations of intervals [5]}

Let $A=\left[a^{L}, a^{U}\right], B=\left[b^{L}, b^{U}\right]$ be two closed intervals in $R$. When $A \geq 0$ and $B \geq 0$ we have:

$$
\begin{aligned}
\text { 1- } & A+B=\left[a^{L}+b^{L}, a^{U}+b^{U}\right] \\
\text { 2- } & A-B=\left[a^{L}-b^{U}, a^{U}-b^{L}\right] \\
\text { 3- } & k A=k\left[a^{L}, a^{U}\right]= \\
& \left\{\begin{array}{l}
{\left[k u^{I}, k a^{I I}\right] \text { if } k \geq 0} \\
{\left[k a^{U}, k a^{L}\right] \text { if } k \leq 0}
\end{array}\right.
\end{aligned}
$$

$$
\begin{aligned}
& \text { 4- } A \times B=\left[a^{L} \times b^{L}, a^{U} \times b^{U}\right] \\
& \text { 5- } A \div B=\left[a^{L} \div b^{U}, a^{U} \div b^{L}\right] .
\end{aligned}
$$

Definition 2.2. Let $A=\left[a^{L}, a^{U}\right], B=\left[b^{L}, b^{U}\right]$ be two closed intervals in $R$. We write $A \leq_{L R} B$ iff $a^{L} \leq b^{L}$ and $a^{U} \leq b^{U}$. Also $A \subseteq_{L R} B$ iff $a^{L} \geq b^{L}$ and $a^{U} \leq b^{U}$ it mean that $A$ is inferior to $B$ or $B$ is superior to $A$.

Definition 2.3. Let $X$ be denote a compact set of real numbers. A rough interval $\mathrm{X}^{R}$ is defined 
as:

$\mathrm{X}^{R}=\left[X^{(L A I)}: X^{(U A I)}\right]$ where $X^{(L A I)}$ and $X^{(U A I)}$

are lower and upper approximation intervals of

$\mathrm{X}^{R}$, respectively with $\mathrm{x}^{(\mathrm{LAI})} \subseteq \mathrm{X}^{(\mathrm{UAI})}$.

Proposition 2.1. For the rough interval

$\mathrm{A}^{R} \in I^{R}$ the following holds:

i. $\mathrm{A}^{K} \geq_{R} 0^{K}$ iff $A^{(L A I)} \geq 0$ and

$$
A^{(U A I)} \geq 0 \text {. }
$$

ii. $\mathrm{A}^{K} \leq_{R} 0^{R}$ iff $A^{(L A I)} \leq 0$ and

$$
A^{(U A D)} \leq 0 \text {. }
$$

Where $I^{R}$ is the set of all rough intervals in $\mathcal{R}$,

$A^{R}=\left[A^{L A I}: A^{U A I}\right]=\left[\left[a^{L L}, a^{U L}\right]:\left[a^{L U}{ }_{3} a^{U U}\right]\right]$,

$a^{L L}, a^{U L}, a^{L U}$ and $a^{U U} \in R$.

\subsection{Basic Operations of Rough Intervals}

For any two rough intervals $A^{R} \geq 0$ and

$B^{R} \geq 0$ we can define the operations on rough

intervals $[7,9,10]$ as follows:

1. Addition:

$A^{\bar{E}} \oplus B^{\bar{E}}=\left[\left[A^{L A I}+B^{L A I}\right]:\left[A^{U A I}+B^{U A I}\right]\right]$

Such that

$\left\{\begin{array}{l}{\left[A^{L A I}+B^{L A I}\right]=\left[a^{\mathrm{L} L}+b^{\mathrm{LL}}, a^{\mathrm{UL}}+b^{\mathrm{UL}}\right],} \\ {\left[A^{U A I}+b^{U A I}\right]=\left[a^{\mathrm{L} U}+b^{\mathrm{L} U}, a^{\mathrm{U} U}+b^{\mathrm{U} U}\right]}\end{array}\right.$

2. Subtraction:

$A^{R} \ominus B^{K}=\left[\left[A^{L A I}-B^{L A I}\right]:\left[A^{U A I}-B^{U A I}\right]\right]$

Such that

$\left\{\begin{aligned}\left\lceil A^{L A I}-B^{L A I}\right\rceil & =\left\lceil a^{L L}-b^{U L}, a^{U L}-b^{L L}\right\rceil, \\ {\left[A^{U A I}-B^{U A I}\right] } & =\left[a^{L U}-b^{U U}, a^{U U}-b^{L U}\right]\end{aligned}\right.$

3. Multiplication:

$A^{K} \circlearrowleft B^{R}=\left[\left[A^{L A I} \times B^{L A I}\right]:\left[A^{U A I} \times B^{U A I}\right]\right]$

Such that

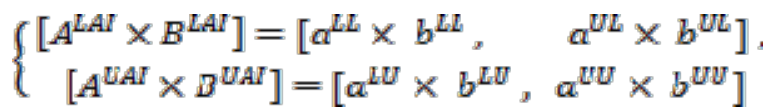

4. Division:

$$
A^{R} \oslash B^{R}=\left[\left[A^{L A I} / B^{L A I}\right]:\left[A^{\text {DAI }} / B^{\text {VAI }}\right]\right]
$$

Such that

$\left\{\begin{array}{c}{\left[A^{L A I} / B^{L A I}\right]=\left[a^{L L} / b^{U L}, a^{U L} / b^{L L}\right]} \\ {\left[A^{U A I} / B^{U A I}\right]=\left[a^{L U} / b^{U U}, a^{U U} / b^{L U}\right]}\end{array}\right.$

Definition 2.4. [7] Let

$A^{R}=\left[\left[a^{L L}, a^{U L}\right]:\left[a^{L U}, a^{U U}\right]\right]$ be in $I^{R}$. Then,

$A^{R}$ is said to be rough integer if

$a^{L L}, a^{U L}, a^{L U}$ and $a^{U U}$ are integers.

\subsection{Integer Linear Fractional Programming Problem}

The general form of integer linear fractional programming (ILFP) problem $[1,2]$ is

discussed as follows:

$\operatorname{Max} \quad Z(x)=\frac{N(x)}{D(x)}=\frac{\sum_{j=1}^{n} c_{j} x_{j}+c_{0}}{\sum_{j=1}^{n} d_{j} x_{j}+d_{0}}$

Subject to:

$\sum_{j=1}^{n} a_{i j} x_{j} \leq b_{i}, i=1_{n} \ldots \ldots m$

$x_{j} \geq 0$ and integers $, j=1, \ldots \ldots n$

where, $c_{j}, d_{j}, c_{0}, d_{0}, a_{i j}$ and $b_{i} \in \mathrm{R}$,

$\sum_{j=1}^{n} d_{j} x_{j}+d_{0} \neq 0$.

\subsection{Variable Transformation Method}

A method is obtained, for solving the linear fractional programming problem with integer variables, through the change of variable $y_{j}=x_{j} t, t>0, \quad$ where $t=\frac{1}{\sum_{j=1}^{n} d_{j} x_{j}+d_{0}}$. The integer linear fractional programming 
problem (1) is transformed into the following problem $[2,11]$ :

$\operatorname{Max} Z(y, t)=\sum_{j=1}^{n} c_{j} y_{j}+c_{0} t$

Subject to:

$\sum_{j=1}^{n} a_{i j} y_{j}-b t \leq 0, \quad i=1, \ldots \ldots, m$

$\left(\sum_{j=1}^{n} d_{j} x_{j}+d_{0}\right) t=1$

$y_{j} \geq 0, t>0$ and $\frac{y_{j}}{t}$ integer $, j=1, \ldots \ldots, n$

Firstly we solve the problem (2) neglecting the condition that $\frac{y_{j}}{t}$ are integers and obtain the solution. If the solution $\frac{y_{j}}{t}$ have all components integer, then it is the optimal solution of the integer linear fractional programming problem (1) except that use the branch and bound method to get the integer solution.

Theorem 2.1. [2] a) If there is an optimal solution of problem (1), then $\left(y^{*}{ }_{3}^{*}\right)$ is an optimal solution of problem (2), where $y^{*}=t^{*} x^{*}$ and $t^{*}=\frac{1}{\sum_{j=1}^{n} d_{j} x_{j}^{*} \| d_{0}}$.

b) Conversely, if there is an optimal solution $\left(y^{*}, t^{*}\right)$ of problem (2), then $t^{*}>0, x^{*}=\frac{y^{*}}{t}$ is an optimal solution of problem (1).

The proof of this theorem is similar with that of theorem (3.4.1) given in [2].

\section{Problem Formulation}

\subsection{Fully rough integer linear fractional} programming (FRILFP) problem

The fully rough integer linear fractional programming problem is defined as follows:
$\operatorname{Max} Z^{R}(x)=\frac{N^{R}(x)}{D^{R}(x)}=\frac{\sum_{j=1}^{n} c_{j}^{R} x_{j}^{R}+c_{0}^{R}}{\sum_{j=1}^{n} d_{j}^{R} x_{j}^{R}+d_{0}^{R}}$

Subject to:

$\sum_{j=1}^{n} A_{i j}^{R} x_{j}^{R} \leq B_{i}^{R}$

$x_{j}{ }^{k} \geq 0$ and rough integer interval

$j=1,2, \ldots, n, i=1,2, \ldots, m$

Where $c_{j}^{R}, d_{j}^{R}, c_{0}^{R}$ and $d_{0}^{R}$ are positive n-vector rough interval defined as:

$c_{j}^{R}=\left[c_{j}^{L}: c_{j}^{U}\right] \quad, \quad d_{j}^{R}=\left[d_{j}^{L}: d_{j}^{U}\right]$,

$c_{0}^{R}-\left[c_{0}^{J_{0}}=c_{0}^{H I}\right] \quad, \quad u_{0}^{R}-\left[d_{0}^{T_{0}}=u_{0}^{I I}\right]$.

$B_{i}^{R}=\left[B_{i}^{L}: B_{i}^{U}\right]$ are $\mathrm{m}$ column and $A_{i j}^{R}=\left[A_{i j}^{L}: A_{i j}^{U}\right]>0$ is an $n \times m$ constraint matrix.

The problem (3) can be written as the form:

$\operatorname{Max} Z^{R}(x)=$

$$
\frac{\left[\sum_{j=1}^{n} c_{j}^{L} x_{j}^{L}+c_{0}^{L}: \sum_{j=1}^{n} c_{j}^{U} x_{j}^{U}+c_{0}^{U}\right]}{\left[\sum_{j=1}^{n} d_{j}^{L} x_{j}^{L}+d_{0}^{L}: \sum_{j=1}^{n} d_{j}^{U} x_{j}^{U}+d_{0}^{U}\right]}
$$

Subject to:

$\sum_{j=1}^{n}\left[A_{i j}^{L}: A_{i j}^{U}\right]\left[x_{j}^{L}: x_{j}^{U}\right] \leq\left[B_{i}^{L}: B_{i}^{U}\right]$

$\left[x_{j}^{L}: x_{j}^{U}\right] \geq 0$ and rough integer interval

$j=1,2, \ldots, n, \quad i=1,2, \ldots, m$

Using the above operations of the rough interval we have:

$\operatorname{Max} Z^{R}(x)=$ $\left[\frac{\sum_{j=1}^{n} c_{j}^{L} x_{j}^{L}+c_{0}^{L}}{\sum_{j=1}^{n} d_{j}^{L} x_{j}^{L}+d_{0}^{L}}: \frac{\sum_{j=1}^{n} c_{j}^{U} x_{j}^{U}+c_{0}^{U}}{\sum_{j=1}^{n} d_{j}^{U} x_{j}^{U}+d_{0}^{U}}\right]$

Subject to: 
$\left[\sum_{j}^{n} A_{i j}^{L} x_{j}^{L}: \sum_{j}^{n} A_{i j}^{U} x_{j}^{V}\right] \leq\left[B_{i}^{L}: B_{i}^{U}\right]$

$\left[x_{j}^{L}: x_{j}^{U}\right] \geq 0$ and rough integer interval

$j=1,2, \ldots, n, i=1,2, \ldots, m$

The fully rough integer linear fractional

programming problem (5) can be written as two integer linear fractional programming problems with interval coefficients $[7,8]$ as follows:

\section{UILFP(1):}

$\operatorname{Max} z^{U}(x)=\begin{aligned} & \sum_{j=1}^{n} c_{j}^{U} x_{j}^{U}+c_{0}^{U} \\ & \sum_{j=1}^{n} d_{j}^{U} x_{j}^{U}+d_{0}^{U}\end{aligned}$

Subject to:

$\sum_{j}^{n} A_{j}^{U} x_{j}^{U} \leq B_{i}^{U}$

$x_{j}^{U} \geq 0$ and integer interval

$j=1,2, \ldots, n, i=1,2, \ldots, m$

\section{LILFP(1):}

$\operatorname{Max} Z^{L}(x)=\frac{\sum_{j=1}^{n} c_{j}^{L} x_{j}^{L}+c_{0}^{L}}{\sum_{j=1}^{n} d_{j}^{L} x_{j}^{L}+d_{0}^{L_{0}}}$

Subject to:

$\sum_{i}^{n} A_{j}^{L} x_{j}^{L} \leq B_{i}^{L}$

$x_{j}^{L} \geq 0$ and integer interval

$j=1, \lambda, \ldots, n . i=1,2, \ldots, m$

\section{LILFP(2):}

$\operatorname{Max}\left[Z^{L L}, Z^{U L}\right]=$

$\frac{\sum_{j=1}^{n}\left[c_{j}^{L L}, c_{j}^{U L}\right]\left[x_{j}^{L L}, x_{j}^{U L}\right]+\left[c_{0}^{L L}, c_{0}^{U L}\right]}{\sum_{j=1}^{n}\left[d_{j}^{L L}, d_{j}^{U L}\right]\left[x_{j}^{L L}, x_{j}^{U L}\right]+\left[d_{0}^{L L}, d_{0}^{U L}\right]}$

Subject to:

$\sum_{j=1}^{n}\left[a_{i j}^{L L}, a_{i j}^{U L}\right]\left[x_{j}^{L L}, x_{j}^{U L}\right] \leq\left[b_{i}^{L L}, b_{i}^{U L}\right]$

$\left[x_{j}^{L L}, x_{j}^{U L}\right] \geq 0$ and integer interval

$j=1,2, \ldots, n, \quad i=1,2, \ldots, m$

Now we know that:

$$
\begin{array}{rlrl}
c_{j}^{L}=\left[c_{j}^{L L}, c_{j}^{U L}\right] & , & c_{j}^{U}=\left[c_{j}^{L U}, c_{j}^{U U}\right], \\
c_{0}^{L}=\left[c_{0}^{L L}, c_{0}^{U L}\right] \quad, & c_{0}^{U}=\left[c_{0}^{L U}, c_{0}^{U U}\right], \\
d_{j}^{L}=\left[d_{j}^{L L}, d_{j}^{U L}\right] \quad, & d_{j}^{U}=\left[d_{j}^{L U}, d_{j}^{U U}\right], \\
d_{0}^{L}=\left[d_{0}^{L L}, d_{0}^{U L}\right] & , & d_{0}^{U}=\left[d_{0}^{L U}, d_{0}^{U U}\right],
\end{array}
$$


$\frac{\sum_{j=1}^{n}\left[c_{j}^{L U} x_{j}^{L U}+c_{0}^{L U}, c_{j}^{U U} x_{j}^{U U}+c_{0}^{U U}\right]}{\sum_{j=1}^{n}\left[d_{j}^{L U} x_{j}^{L U}+d_{0}^{L U}, d_{j}^{U U} x_{j}^{U U}+d_{0}^{U U}\right]}$

Subject to:

$\left[\sum_{1}^{n} a_{i j}^{L U} x_{j}^{L U}, \sum_{1}^{n} a_{i j}^{U U} x_{j}^{U U}\right]<\left[b_{i}^{L U}, b_{i}^{U U}\right]$

$\left[x_{j}^{L U}, x_{j}^{U U}\right] \geq 0$ and integer interval

$j=1,2, \ldots, n, \quad i=1,2, \ldots, m$

\section{LILFP(3):}

$\operatorname{Max}\left[Z^{L L}, Z^{U L}\right]=$

$\frac{\left[\sum_{j-1}^{n} c_{j}^{L L} x_{j}^{L L}+c_{0}^{L L}, \sum_{j-1}^{n} c_{j}^{U L} x_{j}^{U L}+c_{0}^{U L}\right]}{\left[\sum_{j=1}^{n} d_{j}^{L L} x_{j}^{L L}+d_{0}^{L L}, \sum_{j=1}^{n} d_{j}^{U L} x_{j}^{U L}+d_{0}^{U L}\right]}$

Subject to:

$\left[\sum_{1}^{n} a_{i j}^{L L} x_{j}^{L L}, \sum_{1}^{n} a_{i j}^{U L} x_{j}^{U L}\right] \leq\left[b_{i}^{L L}, b_{i}^{U L}\right]$

$\left[x_{j}^{L L}, x_{j}^{U L}\right]>0$ and integer interval

$j=1,2, \ldots, n, i=1,2, \ldots, m$

We can write (10) and (11) as:

\section{UILFP(4):}

$\operatorname{Max}\left[Z^{L U}, Z^{U U}\right]=$

$\left[\frac{\sum_{j=1}^{n} c_{j}^{L U} x_{j}^{L U}+c_{0}^{L U}}{\sum_{j=1}^{n} d_{j}^{U U} x_{j}^{U U}+d_{0}^{U U}}, \frac{\sum_{j=1}^{n} c_{j}^{U U} x_{j}^{U U}+c_{0}^{U U}}{\sum_{j=1}^{n} d_{j}^{L U} x_{j}^{L U}+d_{0}^{L U}}\right]$

Subject to:

$\left[\sum_{1}^{n} a_{i j}^{L U} x_{j}^{L U}, \sum_{1}^{n} a_{i j}^{U U} x_{j}^{U U}\right] \leq\left[b_{i}^{L U}, b_{i}^{U U}\right]$

$\left[x_{j}^{L U}, x_{j}^{U U}\right] \geq 0$ and integer interval

$j=1,2, \ldots, n, \quad i=1,2, \ldots, m$

\section{LILFP(4):}

$\operatorname{Max}\left[Z^{L L}, Z^{U L}\right]=$

$\left[\frac{\sum_{j=1}^{n} c_{j}^{L L} x_{j}^{L L}+c_{0}^{L L}}{\sum_{j=1}^{n} d_{j}^{U L} x_{j}^{U L}+d_{0}^{U L}}, \frac{\sum_{j=1}^{n} c_{j}^{U L} x_{j}^{U L}+c_{0}^{U L}}{\sum_{j=1}^{n} d_{j}^{L L} x_{j}^{L L}+d_{0}^{L L}}\right]$

Subject to:

$\left[\sum_{1}^{n} a_{i j}^{L L} x_{j}^{L L}, \sum_{1}^{n} a_{i j}^{U L} x_{j}^{U L}\right] \leq\left[b_{i}^{L L}, b_{i}^{U L}\right]$

$\left[x_{j}^{L L}, x_{j}^{U L}\right] \geq 0$ and integer interval

$j=1,2, \ldots, n, \quad i=1,2, \ldots, m$

From the above UILFP(4) and LILFP(4) decomposition problem we construct the following four crisp integer linear fractional programming problems named, Upper Upper integer linear fractional programming problem (UUILFP), Upper Lower integer linear fractional programming problem (ULILFP), Lower Lower integer linear fractional programming problem (LLILFP) and Lower Upper integer linear fractional programming problem (LUILFP), as follows:

Model(1): (IIIIII.FP)

$$
\operatorname{Max} Z^{U U}=\frac{\sum_{j=1}^{n} c_{j}^{U U} x_{j}^{U U}+c_{0}^{U U}}{\sum_{j=1}^{n} d_{j}^{I H I} x_{j}^{I H I}+d_{0}^{L U}}
$$

Subject to:

$$
\begin{aligned}
& \sum_{j=1}^{n} a_{i j}^{U U} x_{j}^{U U} \leq b_{i}^{U U} \\
& \sum_{j=1}^{n} a_{i j}^{L U} x_{j}^{L U} \leq b_{i}^{L U} \\
& x_{j}^{L U}, x_{j}^{U U} \geq 0 \text { and integer } \\
& i=1,2, \ldots, n, i=1,2, \ldots, m
\end{aligned}
$$

\section{Model(2): (ULILFP)}


$\operatorname{MaxZ}^{U L}=\frac{\sum_{j=1}^{n} c_{j}^{U L} x_{j}^{U L}+c_{0}^{U L}}{\sum_{j=1}^{n} d_{j}^{L L} x_{j}^{L L}+d_{0}^{L L}}$

Subject to:

$$
\begin{aligned}
& \frac{\sum_{j=1}^{n} c_{j}^{U L} x_{j}^{U L}+c_{0}^{U L}}{\sum_{j=1}^{n} d_{j}^{L L} x_{j}^{L L} \mid d_{0}^{U L}} \leq\left(Z^{U U}\right)^{e} \\
& \sum_{j=1}^{n} a_{i j}^{U L} x_{j}^{U L} \leq b_{i}^{U L} \\
& \sum_{j=1}^{n} a_{i j}^{L L} x_{j}^{L L}<b_{i}^{L L} \\
& x_{j}^{U L} \leq\left(x_{j}^{U U}\right)^{8} \\
& x_{j}^{L L}, x_{j}^{W L} \geq 0 \text { and integer } \\
& j=1,2, \ldots, n, \quad i=1,2, \ldots, m
\end{aligned}
$$

\section{Model(3): (LLILFP)}

$$
\operatorname{Max} Z^{L L}=\frac{\sum_{j=1}^{n} c_{j}^{L L} x_{j}^{L L}+c_{0}^{L L}}{\sum_{j=1}^{n} d_{j}^{U L} x_{j}^{U L}+d_{0}^{U L}}
$$

Subject to:

$$
\begin{aligned}
& \frac{\sum_{j=1}^{n} c_{j}^{L L} x_{j}^{L L}+c_{j}^{U L}}{\sum_{j=1}^{n} d_{j}^{U L} x_{j}^{U L}+d_{0}^{U L}} \leq\left(Z^{U L}\right)^{6} \\
& \sum_{j=1}^{n} x_{i j}^{L L} x_{j}^{L L} \leq h_{i}^{L L} \\
& \sum_{j=1}^{n} a_{i j}^{U L} x_{j}^{U L} \leq b_{i}^{U L} \\
& x_{j}^{L L} \leq\left(x_{j}^{U L}\right)^{e} \\
& x_{j}^{L L}, x_{j}^{U L} \geq 0 \text { and integer } \\
& j-1,2, \ldots, n, i-1,2, \ldots, m
\end{aligned}
$$

\section{Model(4): (LUILFP)}

$\operatorname{Max} Z^{L U}=\frac{\sum_{j=1}^{n} c_{j}^{L U} x_{j}^{L U}+c_{0}^{L U}}{\sum_{j=1}^{n} d_{j}^{U U} x_{j}^{U U}+d_{0}^{U U}}$

Subject to:

$$
\begin{aligned}
& \frac{\sum_{j=1}^{n} c_{j}^{L U} x_{j}^{L U}+c_{0}^{L U}}{\sum_{j=1}^{n} d_{j}^{U U} x_{j}^{U U} d_{0}^{U U}} \leq\left(Z^{L L}\right)^{2} \\
& \sum_{j=1}^{n} a_{i j}^{L U} x_{j}^{L U} \leq b_{i}^{L U} \\
& \sum_{j=1}^{n} a_{i j}^{U U} x_{j}^{U U}<b_{i}^{U U} \\
& x_{j}^{L U} \leq\left(x_{j}^{L L}\right)^{8} \\
& x_{j}^{L U}, x_{j}^{U U} \geq 0 \text { and integer }
\end{aligned}
$$

$$
j=1,2, \ldots, n, \quad i=1,2, \ldots, m
$$

Definition 3.1. A set of rough interval $\left(X_{j}^{R}\right)^{*} \in \mathrm{I}^{\mathbb{R}}$ is said to be an integer optimal solution of the rough integer linear fractional programming (FRILFP) problem if there does not exist $X_{j}^{R} \in \mathrm{I}^{\mathrm{R}}$ such that $Z\left(X_{j}^{R}\right)^{*} \leq Z\left(X_{j}^{R}\right)$.

Theorem 3.1. $[4,11]$ Let

$\left[x_{U U}^{*}\right]=\left\{x_{U V}^{*}: x_{U U}^{*} \in U U\right\}$ be an optimal solution of (UUILFP) problem, $\left[x_{H I}^{*}\right]=\left\{x_{H I}^{*}: x_{H I}^{*} \in L U\right\}$ be an optimal solution of (LUILFP) problem, $\left[x_{U L}^{*}\right]=\left\{x_{U L}^{*}: x_{U L}^{*} \in U L\right\}$ be an optimal solution of (ULILFP) problem, and $\left[x_{L L}^{*}\right]=\left\{x_{L L}^{*}: x_{L L}^{*} \in L L\right\}$ be an optimal solution of (LLILFP) problem, where UU, LU, UL and LL are sets of decision variable in the (UUILFP), (LUILFP), (ULILFP) and (LLILFP) problems respectively. Then the set of rough integer intervals $\left\{\left(X_{j}^{R}\right)^{*}=\left[\left(x_{j}^{L L *}, x_{j}^{U L *}\right):\left(x_{j}^{L U *}, x_{j}^{U U *}\right)\right]\right\}$ is an optimal solution for the given (FRILFP) problem .

Proof: Let

$\left\{\left(Y_{j}^{R}\right)=\left[\left(y_{j}^{L L}, y_{j}^{U L}\right):\left(y_{j}^{L U}, y_{j}^{U U}\right)\right]\right.$ for all $j \in$ I\}

be a feasible solution to the (FRILFP) problem.

Clearly, $\left[y_{U U}\right],\left[y_{L U}\right],\left[y_{U L}\right]$ and $\left[y_{L L}\right]$ are feasible solution to the problems (UUILFP), (LUILFP), (ULILFP) and (LLILFP) respectively.

Now, since $\left[x_{U U}^{*}\right],\left[x_{L U}^{*}\right],\left[x_{U L}^{*}\right]$, and $\left[x_{L L}^{*}\right]$ are optimal solution for the problems (UUILFP), 
(LUILFP), (ULILFP) and (LLILFP)

respectively, we have

$Z^{U U}\left(\left[x_{U U}^{*}\right]\right) \leq Z^{U U}\left(\left[y_{U U}\right]\right)$

$Z^{L U}\left(\left[x_{L U}^{*}\right]\right) \leq Z^{L U}\left(\left[y_{L U}\right]\right)$,

$Z^{U L}\left(\left[x_{U L}^{*}\right]\right) \leq Z^{U L}\left(\left[v_{U L}\right]\right)$ and

$Z^{L L}\left(\left[x_{L L}^{*}\right]\right) \leq Z^{L L}\left(\left[\gamma_{L L}\right]\right)$.

This implies that $Z\left(X_{j}^{R *}\right) \leq Z\left(Y_{j}^{R}\right)$, for all feasible solution of the (FRILFP) problem.

Therefore, the set of rough integer intervals

$\left\{\left(X_{j}^{R}\right)^{*}=\left[\left(x_{j}^{L L *}, x_{j}^{U L *}\right):\right.\right.$

$\left.\left(x_{j}^{L U *}, x_{j}^{U U *}\right)\right]$, for all $\left.j \in J\right\}$

is an optimal solution for the (FRILFP)

problem. Hence, the theorem is proved.

\section{Algorithm: Solution for FRILFP problem}

The propose algorithm to solve (FRILFP)

problem can be summarized in the following steps:

Step 1. Use the given problem to construct four crisp integer linear fractional programming problem namely Upper

Upper integer linear fractional programming problem, Upper Lower integer linear fractional programming problem, Lower Lower integer linear fractional programming problem and Lower Upper integer linear fractional programming problem.

Step 2. Find the integer optimal solution $\left(x_{j}^{U U}\right)^{*}$ for (UUILFP) problem with the objective value $\left(Z^{U U}\right)^{8}$, by the variable transformation method.

Step 3. Solve the (ULILFP) problem by the variable transformation method to obtain the integer optimal solution $\left(x_{j}^{U L}\right)^{s}$ with the objective value $\left(Z^{U L}\right)^{e}$.

Step 4. Solve the (LLILFP) problem by the variable transformation method to obtain the integer optimal solution $\left(x_{j}^{L L}\right)^{\varepsilon}$ with the objective value $\left(Z^{L L}\right)^{\varepsilon}$.

Step 5. Solve the (LUILFP) problem by the variable transformation method to obtain the integer optimal solution $\left(x_{j}^{L U}\right)^{g}$ with the objective value $\left(Z^{L U}\right)^{*}$

Step 6. The set of rough integer interval $\left(X_{j}^{R}\right)^{*}=\left[\left(x_{j}^{L L *}, x_{j}^{U L *}\right):\right.$ $\left.\left(x_{j}^{L U *}, x_{j}^{U U_{*}}\right)\right]$ is an integer optimal solution to the given problem (FRILFP) with the objective value $\left(Z^{R}\right)^{*}=\left[\left(Z^{L L^{*}}, Z^{U L^{*}}\right):\right.$
$\left.\left(Z^{L U^{*}}, Z^{U U^{*}}\right)\right]$
by the theorem (3.1).

\section{Numerical example}

Consider the following (FRILFP) problem:

$\operatorname{Max} Z^{R}(x)=$

([3,6]: $[2,8]) X_{1}^{R}+([6,8]:[4,11]) X_{2}^{R}$

$([4,7]:[3,8]) X_{1}^{R}+([3,5]:\lceil 2,6]) X_{2}^{R}+([6,10] ;[4,13])$

$([1,3]:[1,4]) X_{1}^{R}+([2,5]:[1,6]) X_{2}^{R}$

$$
\leq([20,30]:[18,55])
$$

$([4,7]:[2,8]) X_{1}^{R}+([1,3]:[1,4]) X_{2}^{R}$

$$
\leq([25,27] \cdot[20,50])
$$

$X_{1}^{R}, X_{2}^{R} \geq 0$ and rough integer intervals 
Where

$X_{1}^{R}=\left[X_{1}^{L}: X_{1}^{U}\right]=\left[\left(x_{1}^{L L}, x_{1}^{L L}\right):\left(X_{1}^{L U}, X_{1}^{U U}\right)\right]$

$X_{2}^{R}=\left[X_{1}^{L}: X_{1}^{U}\right]=\left[\left(x_{2}^{L L}, x_{2}^{U L}\right):\left(x_{2}^{L U}, x_{2}^{U U}\right)\right]$

Solution: Firstly we can write the problem on the form

$\operatorname{Max} Z^{R}(x)=$

$\left[\frac{[3,6] X_{1}^{L}+[6,8] X_{2}^{L}:[2,8] X_{1}^{U}+[4,11] X_{2}^{U}}{[4,7] X_{1}^{L}+[3,5] X_{2}^{L}+[6,10]:[3,8] X_{1}^{U}+[2,6] X_{2}^{U}+[4,13]}\right]$

Subject to:

$\left[[1,3] X_{1}^{L}+[2,5] X_{2}^{L}:[1,4] X_{1}^{U}+[1,6] X_{2}^{U}\right]$ $\leq([20,30]:[18,55])$

$\left[[4,7] X_{1}^{L}+[1,3] X_{2}^{L}:[2,8] X_{1}^{U}+[1,4] X_{2}^{U}\right]$

$$
\leq([25,27]:[20,50])
$$

$\left[x_{1}^{L}: x_{1}^{U}\right],\left[x_{2}^{L}: x_{2}^{U}\right] \geq 0$ and rough integer

Using the arithmetic operations we have

$\operatorname{MaxZ}^{R}(x)=$

$\left\lfloor\frac{[3,6] X_{1}^{L}+[6,8] X_{2}^{L}}{[4,7] X_{1}^{L}+[3,5] X_{2}^{L}+[6,10]}: \frac{[2,8] X_{1}^{U}+[4,11] X_{2}^{U}}{[3,8] X_{1}^{U}+[2,6] X_{2}^{U}+[4,13]}\right\rfloor$

Subject to:

$$
\begin{array}{r}
{\left[[1,3] x_{1}^{L}+[2,5] x_{2}^{L}:[1,4] X_{1}^{U}+[1,6] X_{2}^{U}\right]} \\
\leq([20,30]:[18,55]) \\
{\left[[4,7] x_{1}^{L}+[1,3] x_{2}^{L}:[2,8] X_{1}^{U}+[1,4] X_{2}^{U}\right]} \\
\leq([25,27]:[20,50])
\end{array}
$$

$\left[x_{1}^{L_{0}}: x_{1}^{S I}\right],\left[x_{2}^{I_{D}} ; x_{2}^{I I}\right] \geq 0$ and rough integer

Now we will divide this problem into two integer interval linear fractional programming problems $[7,8]$ as the following:

$\operatorname{UILFP}(1): \quad \operatorname{Max} Z^{U}(x)=$

$$
\frac{[2,8]\left[X_{1}^{L U},{ }_{,} X_{1}^{U U}\right]+[4,11]\left[X_{2}^{L U},{ }_{,} X_{2}^{U U}\right]}{[3,8]\left[X_{1}^{L U}{ }_{,} X_{1}^{U U}\right]+[2,6]\left[X_{2}^{L U},{ }^{L U} X_{2}^{U U}\right]+[4,13]}
$$

Subject to:

$$
[1,4]\left[X_{1}^{L U}, X_{1}^{U U}\right]+[1,6]\left[X_{2}^{L U}, X_{2}^{U U}\right] \leq[18,55]
$$

$[2,8]\left[X_{1}^{L U}, X_{1}^{U U}\right]+[1,4]\left[X_{2}^{L U}, X_{2}^{L U}\right] \leq[20,50]$

$\left[X_{1}^{L U}, X_{1}^{U U}\right],\left[X_{2}^{L U}, X_{2}^{U U}\right] \geq 0$ and integer

$\operatorname{LILFP(1):~} \quad \operatorname{Max} Z^{L}(x)=$

$$
\frac{[3,6]\left[X_{1}^{L L}, X_{1}^{U L}\right]+[6,8]\left[X_{2}^{L L}, X_{2}^{U L}\right]}{[4,7]\left[X_{1}^{L L}, X_{1}^{U L}\right]+[3,5]\left[X_{2}^{L L}, X_{2}^{U L}\right]+[6,10]}
$$

Subject to:

$$
\begin{gathered}
{[1,3]\left[X_{1}^{L L}, X_{1}^{U L}\right]+[2,5]\left[X_{2}^{L L}, X_{2}^{U L}\right] \leq[20,30]} \\
{[4,7]\left[X_{1}^{L L}, X_{1}^{U L}\right]+[1,3]\left[X_{2}^{L L}, X_{2}^{U L}\right] \leq[25,27]} \\
{\left[X_{1}^{L L}, X_{1}^{U L}\right],\left[X_{2}^{L L}, X_{2}^{U L}\right] \geq 0 \text { and integer }}
\end{gathered}
$$

The problems UILFP(1)and LILFP(1) can be written as:

$\operatorname{ULLFP(Z):} \quad \operatorname{MaxZ}^{U}(x)=$

$$
\frac{\left[2 X_{1}^{L U}+4 X_{2}^{L U}, 8 X_{1}^{U U}+11 X_{2}^{U U}\right]}{\left[3 X_{1}^{I I T}+2 X_{2}^{I H}+4,0 X_{1}^{I W}+6 X_{2}^{W W}+13\right]}
$$

Subject to:

$$
\begin{aligned}
& {\left[X_{1}^{L U}+X_{2}^{L U}, 4 X_{1}^{U U}+6 X_{2}^{U U}\right] \leq[18,55]} \\
& {\left[2 X_{1}^{L U}+X_{2}^{L U}, 8 X_{1}^{U U}+4 X_{2}^{U U}\right] \leq[20,50]} \\
& {\left[X_{1}^{L U}, X_{1}^{U U}\right],\left[X_{2}^{L U}, X_{2}^{U U}\right] \geq 0 \text { and integer }}
\end{aligned}
$$

$$
\text { LILFP(2): } \quad \operatorname{MaxZ}^{L}(x)=
$$

$$
\frac{\left[3 X_{1}^{L L}+6 X_{2}^{L L}, 6 X_{1}^{U L}+8 X_{2}^{U L}\right]}{\left[4 X_{1}^{L L}+3 X_{2}^{L L}+6,7 X_{1}^{U L}+5 X_{2}^{U L}+10\right]}
$$

Subject to:

$$
\begin{aligned}
& {\left[X_{1}^{L L}+2 X_{2}^{L L}, 3 X_{1}^{U L}+5 X_{2}^{U L}\right] \leq[20,30]} \\
& {\left[4 X_{1}^{L L}+X_{2}^{L L}, 7 X_{1}^{U L}+3 X_{2}^{U L}\right] \leq[25,27]} \\
& {\left[X_{1}^{L L}, X_{1}^{U L}\right],\left[X_{2}^{L L}, X_{2}^{U L}\right] \geq 0 \text { and integer }}
\end{aligned}
$$

Using the arithmetic operation of interval we get four crisp integer linear fractional programming problem as the following:

\section{Model(1): UUILFP}

$\operatorname{Max} Z^{U U}(x)=\frac{8 X_{1}^{U U}+11 X_{2}^{U U}}{3 X_{1}^{L U}+2 X_{2}^{L U}+4}$ 
Subject to:

$X_{1}^{L U}+X_{2}^{L U} \leq 18,2 X_{1}^{L U}+X_{2}^{L U} \leq 20$

$4 X_{1}^{U U}+6 X_{2}^{U U} \leq 55,8 X_{1}^{U U}+4 X_{2}^{U U} \leq 50$

$X_{1}^{L U}, X_{1}^{U U}, X_{2}^{L U}, X_{2}^{U U} \geq 0$ and integers

Solving the (UUILFP) problem by variable transformation method ignoring the integrality condition we have

$X_{1}^{L U}=0, X_{1}^{U U}=2.5, X_{2}^{L U}=0, X_{2}^{U U}=7.5$ and $Z^{U U}\left(x^{*}\right)=25.625$, Since the decision

variables are not all integer, then Apply branch and bound method to get an integer optimal solution:

$X_{1}^{L U}=0, X_{1}^{U U}=0, X_{2}^{L U}=0, X_{2}^{U U}=9$, $Z^{U U}\left(x^{*}\right)=24.75$

\section{Model(2): ULILFP}

$\operatorname{Maxz}^{U L}(x)-\frac{6 X_{1}^{U L}+8 X_{2}^{U L}}{4 X_{1}^{L L}+3 X_{2}^{L L}+6}$

Subject to:

$$
\begin{gathered}
\frac{6 X_{1}^{U L}+8 x_{2}^{U L}}{2 x_{1}^{L L}+3 x_{2}^{L L}+6} \leq 24.75 \\
X_{1}^{L L}+2 X_{2}^{L L} \leq 20,4 X_{1}^{L L}+X_{2}^{L L} \leq 25 \\
3 X_{1}^{U L}+5 X_{2}^{U L} \leq 30,7 X_{1}^{U L}+3 X_{2}^{U L} \leq 27 \\
X_{1}^{U L} \leq 0, \quad X_{2}^{U L} \leq 9 \\
X_{1}^{L L}, X_{1}^{U L}, X_{2}^{L L}, X_{2}^{U L} \geq 0 \text { and integers }
\end{gathered}
$$

Now, Using the variable transformation method to solve the (ULILFP) problem without integer condition to get the following results:

$X_{1}^{L L}=0, \quad Z_{2}^{L L}=0, \quad X_{1}^{U L}=0, \quad X_{2}^{U L}=6$ and $Z^{U L}\left(x^{8}\right)=8$.

Since the decision variables are all integer, then the integer optimal solution is:

$X_{1}^{L L}=0, \quad X_{2}^{L L}=0, \quad X_{1}^{U L}=0, \quad X_{2}^{U L}=6$ and $z^{U L}\left(x^{8}\right)=8$.

\section{Model(3): LLILFP}

$\operatorname{Max} Z^{L L}(x)=\frac{3 X_{1}^{L L}+6 X_{2}^{L L}}{7 X_{1}^{I I I_{0}}+5 X_{2}^{I I I_{0}}+10}$

Subject to:

$$
\begin{gathered}
\frac{3 X_{1}^{L L}+6 X_{2}^{L L}}{7 X_{1}^{I L L}+5 X_{2}^{I I D}+10} \leq 8 \\
X_{1}^{L L}+2 X_{2}^{L L} \leq 20, \quad 4 X_{1}^{L L}+X_{2}^{L L} \leq 25 \\
3 X_{1}^{U L}+5 X_{2}^{U L} \leq 30, \quad 7 X_{1}^{U L}+3 X_{2}^{U L} \leq 27 \\
X_{1}^{L L} \leq 0, \quad X_{2}^{L L} \leq 6 \\
X_{1}^{L L}, X_{1}^{U L}, X_{2}^{L L}, X_{2}^{U L} \geq 0 \text { and integers }
\end{gathered}
$$

Now, substituting $\quad X_{1}^{L L}=0, \quad X_{2}^{L L}=0$, $X_{1}^{U L}=0, \quad X_{2}^{U L}=6$ in the (LLILFP)

problem, the integer optimal solution is

$X_{1}^{L L}=0, \quad X_{2}^{L L}=0, \quad X_{1}^{U L}=0, \quad X_{2}^{U L}=6$ and $Z^{U L}\left(x^{8}\right)=0$.

\section{Model(4): LUILFP}

$\operatorname{Max} Z^{L U}(x)=\frac{2 X_{1}^{L U}+4 X_{2}^{L U}}{8 X_{1}^{U U}+6 X_{2}^{U U}+13}$

Subject to:

$$
\begin{gathered}
\frac{2 X_{1}^{L U}+4 X_{2}^{L U}}{8 X_{1}^{U U}+6 X_{2}^{U U}+13} \leq 0 \\
X_{1}^{L U} \mid X_{2}^{L U} \leq 18, \quad 2 X_{1}^{L U} \| X_{2}^{L U} \leq 20 \\
4 X_{1}^{U U}+6 X_{2}^{U U} \leq 55, \quad 8 X_{1}^{U U}+4 X_{2}^{U U} \leq 50 \\
X_{1}^{L U} \leq 0, \quad X_{2}^{L U} \leq 0 \\
X_{1}^{L U}, X_{1}^{U U}, X_{2}^{L U}, X_{2}^{U U} \geq 0 \text { and integers }
\end{gathered}
$$

Now, substituting $\quad X_{1}^{L U}=0, X_{2}^{L U}=0$, $X_{1}^{U U}=0, \quad X_{2}^{U U}=9 \quad$ in the (LUILFP) problem, the integer optimal solution is $X_{1}^{L U}=0, X_{2}^{L U}=0, X_{1}^{U U}=0, \quad X_{2}^{U U}=9$ and $Z^{U L}\left(x^{*}\right)=0$.

Therefore, by theorem (3.1) the rough integer optimal solution for the given (FRILFP) problem is $\left(x_{1}^{R}\right)^{*}=\lfloor[0,0]:[0,0]\rfloor$, 
$\left(x_{2}^{R^{R}}\right)^{*}=[[0,6]:[0,9]]$ with the maximum

objective value $Z^{R}\left(x^{*}\right)-[[0,8] ;[0,24.75]]$.

\section{Conclusion}

In this paper, we focused on the solution of the fully rough integer linear fractional programming (FRILFP) problem, where all decision variables and coefficients are rough intervals. The proposed approach was based on operations of intervals, operations of rough interval, variable transformation method and brunch and bound method to get an integer optimal solution. Finally, a numerical example is given for the sake of illustration.

\section{References}

1. Sharma, S. and A. Bansal, An integer solution of fractional programming problem. General Mathematics Notes, 2011. 4(2): p. 1-9.

2. Stancu-Minasian, I., Fractional programming: theory, methods and applications. 1997, Dordrecht: Kluwer Academic Pubisher.

3. Borza, M., A.S. Rambely, and M. Saraj, Solving linear fractional programming problems with interval coefficients in the objective function. A new approach. Applied Mathematical Sciences, 2012. 6(69-72): p. 3443-3459.

4. Ponnaiah, P. and J. Mohan, On solving linear fractional programming problems. Modern Applied Science, 2013. 7(6): p. 90-100.

5. Effati, S. and M. Pakdaman, Solving the interval-valued linear fractional programming problem. American

Journal of Computational Mathematics, 2012. 2(01): p. 51.

6. Pawlak, Z., Rough sets. International journal of computer \& information sciences, 1982. 11(5): p. 341-356.

7. Pandian, P., G. Natarajan, and A. Akilbasha, Fully rough integer interval transportation problems. International Journal Of Pharmacy and Technology, 2016. 8: p. 13866-13876.

8. Hamzehee, A., M.A. Yaghoobi, and M. Mashinchi, Linear programming with rough interval coefficients. Journal of Intelligent \& Fuzzy Systems, 2014. 26(3): p. 1179-1189.

9. Ammar, E. and M. Muamer, Solving a rough linear fractional programming problem. Journal of Advances in Mathematics 2015. 10(4): p. 33743380 .

10. Emam, O., E. Fathy, and A. Abohany, An Interactive Model for Fully Rough

Three Level Large Scale Integer Linear Programming Problem. International Journal of Computer Applications, 2016. 155(12): p. 1-12.

11. Ammar, E. and M. Muamer, On Solving Fuzzy Rough Linear Fractional Programming Problem. International Research Journal of Engineering and Technology (IRJET), 2016. 3(4): p. 2099-2120.

12. Omran, M., et al., Solving Large-scale Three-level Linear Fractional 
Programming Problem with Rough

Coefficient in Objective Function.

International Journal of Computer

Applications, 2017. 157(8): p. 25-29. 